\title{
A qualitative description of microstructure formation and coarsening phenomena for an evolution equation
}

\author{
Tomás Caraballo and Renato Colucci
}

\begin{abstract}
We provide a qualitative description of microstructure formation and coarsening phenomena for the solutions of a singularly perturbed fourth order evolution equation arising in the study of phase transitions. In particular we study stationary and traveling wave solutions and we construct a class of approximate solution which mimics the principle features of the dynamics. Finally we present several simulations in order to illustrate the results.
\end{abstract}

Mathematics Subject Classification (2010). Primary 35B36, 35B10, 35B25, 35B40; Secondary 82C26.

Keywords. Forward-Backward Diffusion, Phase Transitions, Traveling waves.

\section{Introduction}

Forward-backward equations ([12], [18] and references cited therein) arise in many contexts such as nonlinear elasticity ([15]), image processing and phase transitions, the interest of researchers in this problem is motivated by the rich observed phenomenology. The evolution equation

$$
u_{t}=W^{\prime \prime}\left(u_{x}\right) u_{x x},
$$

is ill-posed due to the change of sign of the diffusion coefficient (see [6]), or analogously, the corresponding minimum problem for the energy

$$
F(u)=\int_{I} W\left(u_{x}\right) d x
$$

admits infinite many minimizers. Here, the function $W(p)=\left(p^{2}-1\right)^{2}$ is the so called double well potential and $I=(0,1)$.

This work has been partially supported by FEDER and the Spanish Ministerio de Economía y Competitividad project MTM2015-63723-P and the Consejería de Innovación, Ciencia y Empresa (Junta de Andalucía) under Proyecto de Excelencia P12-FQM-1492. 
In general it is convenient to consider a regularized version of the problem, such as

$$
u_{t}+\varepsilon^{2} u_{x x x x}=W^{\prime \prime}\left(u_{x}\right) u_{x x},
$$

where $0<\varepsilon<<1$. If the parameter $\varepsilon$ is sufficiently small, it is natural to expect that we have a good approximation of the original problem. Equation (1.2) has been studied in [1] and [2], where the authors pointed out the existence of three well separated time scales with different dynamical behavior. In a first time scale of order $\mathcal{T}_{\varepsilon}=O\left(\varepsilon^{2}\right)$ they observed the formation of microstructure (see [14]) of spatial wave length of order $O(\varepsilon)$. Microstructure appears in regions where the first derivative of the initial datum $u_{0}$ falls in the non convex region of the potential $W(\cdot)$ (or equivalently in the region in which the diffusion coefficient of (1.1) is negative).

In the second time scale of order $O(1)$,regions without microstructure exhibit heat equation-like behavior. The separation between regions with and without microstructures may give rise to a free boundary problem. In the third time scale of order $\mathcal{T}_{\varepsilon}^{-1}=O\left(\varepsilon^{-2}\right)$, the equation exhibits a finite dimensional behavior, the solutions are approximately the union of segments with slopes \pm 1 and the number of degrees of freedom for the system is approximately represented by the number of jumps of $u_{x}$. This time scale is characterized by very slow motion and the number of jumps decreases very slowly giving rise to the so called coarsening phenomenon.

In this paper we consider the third time scale, that is the long term behavior. This is well described by the global attractor $\mathcal{A}([19])$, in the sense that any fixed trajectory, for a large time, is well approximated by portions of trajectories which lie in the attractor (see [16], corollary 10.15 pag. 277, termed as tracking property). For this reason, it is very interesting to study the structure of the global attractor and the dynamics on it. For gradient systems, such as (1.2), whose Lyapunov function is given by

$$
F_{\varepsilon}(u)=\frac{1}{2} \varepsilon^{2} \int_{I} u_{x x}^{2} d x+\frac{1}{2} \int_{I} W\left(u_{x}\right) d x
$$

it is well known (see [16]) that the attractor is given by the unstable manifold of the set $\mathcal{E}=\mathcal{E}(\varepsilon)$ of stationary solutions:

$$
\mathcal{A}=W^{u}(\mathcal{E})
$$

if $\mathcal{E}$ is discrete we have an even simpler expression:

$$
\mathcal{A}=\bigcup_{z \in \mathcal{E}} W^{u}(z)
$$

Unfortunately, in our case, we cannot conclude that the set $\mathcal{E}$ is discrete. However, we still consider interesting enough to study the set $\mathcal{E}$ in order to obtain some information on the structure of the attractor and, as a consequence, of the long term behavior of the solutions of (1.2).

In [3]-[10] the problem of microstructure formation and finite dimension dynamics have been studied. In particular, the following upper bound of the 
fractal dimension of the global attractor $\mathcal{A}_{\varepsilon}$ of (1.2) has been obtained (together with the existence and dimension estimate of exponential attractor, [13] and inertial manifold, [11] ):

$$
d_{F}\left(\mathcal{A}_{\varepsilon}\right) \leq O\left(\varepsilon^{-1}\right)
$$

where $d_{F}(X)$ is the fractal dimension of $X$ which is based on counting the number of ball of a fixed radius that are necessary to cover $X$ (see [16], [17] for a detailed discussion).

This is in accordance with what we expected by looking at the numerical experiments and, in particular, the estimate of the spatial wave length of microstructure. In fact, as the degrees of freedom of the system are well represented by the number of jumps $J_{x}$ of $u_{x}$, we obtain an upper bound of their number by

$$
J_{x} \approx \frac{|I|}{\varepsilon}=O\left(\varepsilon^{-1}\right)
$$

(see [7] for a more detailed discussion).

In Section 2 below we will prove that it is possible to recover many of these ideas by studying some properties of stationary solutions. Among the general solutions of (1.2) we decided to dedicate some effort to the study of traveling waves on the whole $\mathbb{R}$, since this will allow us to describe some of the previously mentioned phenomenology. In Section 3 we deal with traveling wave analysis, we will see that, in full accordance with the above cited corollary in [16], these kind of solutions will be very close to stationary periodic solutions for a time of order smaller than $O\left(\varepsilon^{2}\right)$, while for time of order $O\left(\varepsilon^{-2}\right)$ we have that $u_{x}$ goes to 1 ( -1 respectively) as $(x-c t) \rightarrow \infty$, and $u$ will be close to a class of unbounded solutions associated to heteroclinic connections in the plane $\left(u_{x}, u_{x x}\right)$ (see figure 3 below). This means that $u_{x}$ starts to loose its oscillatory behavior and converges to \pm 1 as expected by the observed coarsening phenomenon.

In Section 4 we construct an approximate solution which will describe what has been obtained in Section 3. Moreover, we present several numerical simulations to illustrate the results.

\section{Stationary Solutions}

In this section we study some properties of the stationary solutions of equation (1.2) in order to have some qualitative description of the dynamics. A more complete study of the set of stationary solutions is postponed for future investigation. In details we look for the solutions of

$$
\varepsilon^{2}\left(u_{x x x x}\right)=\frac{1}{2}\left[W^{\prime}\left(u_{x}\right)\right]_{x}
$$

which can be rewritten, after integration, in the following form

$$
\varepsilon^{2} u_{x x x}=\frac{1}{2} W^{\prime}\left(u_{x}\right)+K,
$$


where $K$ is a real constant.

We rewrite the above third order ordinary differential equation as a first order three dimensional system by using the usual identification

$$
u=x_{1}, \quad u_{x}=x_{2}, \quad u_{x x}=x_{3},
$$

obtaining

$$
\left\{\begin{array}{l}
\dot{x_{1}}=x_{2} \\
\dot{x_{2}}=x_{3} \\
\dot{x_{3}}=\frac{1}{\varepsilon^{2}}\left\{2 x_{2}\left(x_{2}^{2}-1\right)+K\right\} .
\end{array}\right.
$$

If $K \neq 0$ the system has no fixed points. We note that in this case, by integrating the quotient of the last two equations, we obtain

$$
\frac{1}{2} x_{2}^{4}-x_{2}^{2}+K x_{2}+S=\frac{1}{2}\left(x_{2}^{2}-1\right)^{2}+K x_{2}+S-\frac{1}{2}=\frac{1}{2} \varepsilon^{2} x_{3}^{2},
$$

where $S$ is another real constant. For simplicity we rewrite the previous expression in the following way:

$$
\left(x_{2}^{2}-\frac{3}{2}\right)^{2}+\left(x_{2}+K\right)^{2}+\left(2 S-K^{2}-\frac{9}{4}\right)=\varepsilon^{2} x_{3}^{2} .
$$

We note that, if $S>\frac{K^{2}}{2}+\frac{9}{8}$, the previous expression is defined for all $x_{2} \in \mathbb{R}$ then there are no periodic orbits in the plane $x_{2} x_{3}$. In figure 2 below we represent the level curves of the difference between the r.h.s. and the 1.h.s. of (2.4) for a fixed value of $K$ and several values of $S$. We observe the existence of a region with periodic orbits (see figure 2) on the plane $x_{2} x_{3}$.

For example if $K=\frac{1}{2}$ and $S=0$ we have

$$
x_{2}\left(x_{2}-1\right)\left(x_{2}^{2}+x_{2}-1\right)=\varepsilon^{2} x_{3}^{2},
$$

which is defined if $x_{2}<\frac{-1-\sqrt{5}}{2}$, if $x_{2} \in\left[0, \frac{-1+\sqrt{5}}{2}\right]$ or if $x_{2}>1$. In particular the periodic orbit correspond to the interval $x_{2} \in\left[0, \frac{-1+\sqrt{5}}{2}\right]$. We have $x_{3}=0$ for $x_{2} \in\left\{0, \frac{-1+\sqrt{5}}{2}\right\}$ while the maximum of $x_{3}$ is obtained at (see figure 1 below)

$$
4 x_{2}\left(x_{2}^{2}-1\right)+1=0, \quad \text { that is } x_{2} \approx 0.26 .
$$

Remark 2.1. A periodic orbit in the $\left(x_{2}, x_{3}\right)$ plane corresponds to an oscillatory stationary solution, that is, a solution with both oscillatory first and second derivatives.

If $K=0$ we have a line of fixed points for the three dimensional system:

$$
l=\{(x, 0,0): \quad x \in \mathbb{R}\},
$$

and the Jacobian of the system at any point of the line $l$ is given by

$$
\left(\begin{array}{ccc}
0 & 1 & 0 \\
0 & 0 & 1 \\
0 & -\frac{2}{\varepsilon^{2}} & 0
\end{array}\right)
$$

with trace and determinant

$$
\operatorname{Tr}(J)=0, \quad \operatorname{Det}(J)=0 .
$$




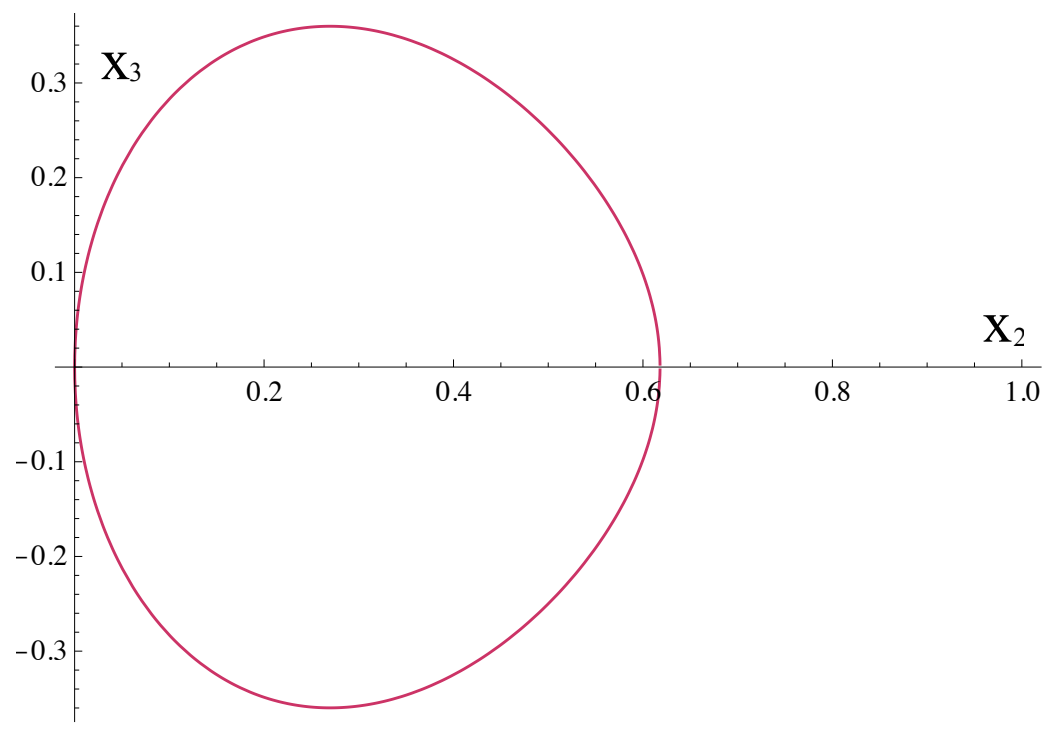

Figure 1. The periodic orbit for $K=\frac{1}{2}$ and $S=0$.

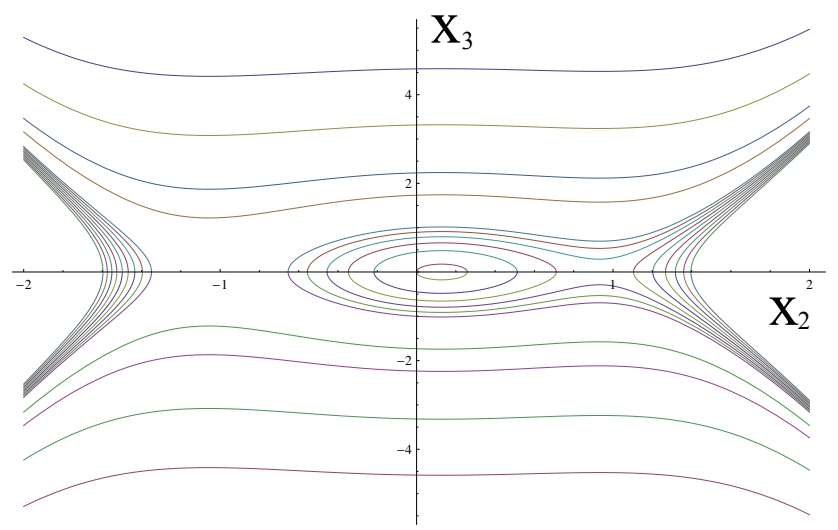

Figure 2. The orbits $\left(x_{2}, x_{3}\right)$ for $K=\frac{1}{4}$, several values of $S$ and $\varepsilon=1$.

We consider the following change of variables in order to deduce the canonical form for the Jacobian of the system:

$$
x_{1}=X, \quad x_{2}=Y, \quad x_{3}=\frac{\sqrt{2}}{\varepsilon} Z .
$$


Then the system takes the form:

$$
\left(\begin{array}{c}
\dot{X} \\
\dot{Y} \\
\dot{Z}
\end{array}\right)=\left(\begin{array}{ccc}
0 & 1 & 0 \\
0 & 0 & \frac{\sqrt{2}}{\varepsilon} \\
0 & -\frac{\sqrt{2}}{\varepsilon} & 0
\end{array}\right)\left(\begin{array}{c}
X \\
Y \\
Z
\end{array}\right)+\left(\begin{array}{c}
0 \\
0 \\
\frac{\sqrt{2}}{\varepsilon} Y^{3}
\end{array}\right),
$$

and the Jacobian at any point of $l$ is

$$
\left(\begin{array}{ccc}
0 & 1 & 0 \\
0 & 0 & \frac{\sqrt{2}}{\varepsilon} \\
0 & -\frac{\sqrt{2}}{\varepsilon} & 0
\end{array}\right)
$$

Of course we have again zero trace and determinant, while the eigenvalues are

$$
\lambda_{1}=0, \quad \lambda_{2,3}=i \frac{\sqrt{2}}{\varepsilon}:=i \omega .
$$

We note that the second and third equations of system (2.6), do not depend on $X$. Moreover the system restricted on the plane $(Y, Z)$ can be written in Hamiltonian form:

$$
\left\{\begin{array}{l}
\dot{Y}=\frac{\partial H}{\partial Z}, \\
\dot{Z}=-\frac{\partial H}{\partial Y} .
\end{array}\right.
$$

with the first integral (see figure 3 below)

$$
H(Y, Z)=\frac{1}{\sqrt{2} \varepsilon}\left[-\frac{1}{2}\left(Y^{2}-1\right)^{2}+Z^{2}\right] .
$$

The projection of solutions on the $Y Z$ plane are the level curves of the function $H$ (see figure 3 below).

We note that if we are looking for bounded solutions, we need to restrict the analysis to the case in which the system on the plane $Y Z$ possesses bounded solutions.

In general we have the following result:

Theorem 2.2. System (2.6) admits the following classes of solutions depending on the values of (2.9).

1. If $H>0$, there exist unbounded solutions in the $Y Z$ plane. As a consequence, the $X$ component is unbounded too.

2. If $H=0$ it follows that:

$\diamond$ If $\left(Y_{0}, Z_{0}\right)=( \pm, 1,0)$, which correspond to the fixed points of the $Y Z$ system, we obtain the following unbounded solutions:

$$
(X, Y, Z)=\left(X_{0} \pm t, \pm 1,0\right) \text {. }
$$

$\diamond$ The heteroclinic connections which connect the fixed points $( \pm 1,0)$ of the $Y Z$ system correspond to unbounded solutions of the three dimensional system since $Y$ remains near \pm 1 asymptotically and as a consequence $X \rightarrow \pm \infty$.

$\diamond$ There exist unbounded solutions for $\left|Y_{0}\right|>1$. 


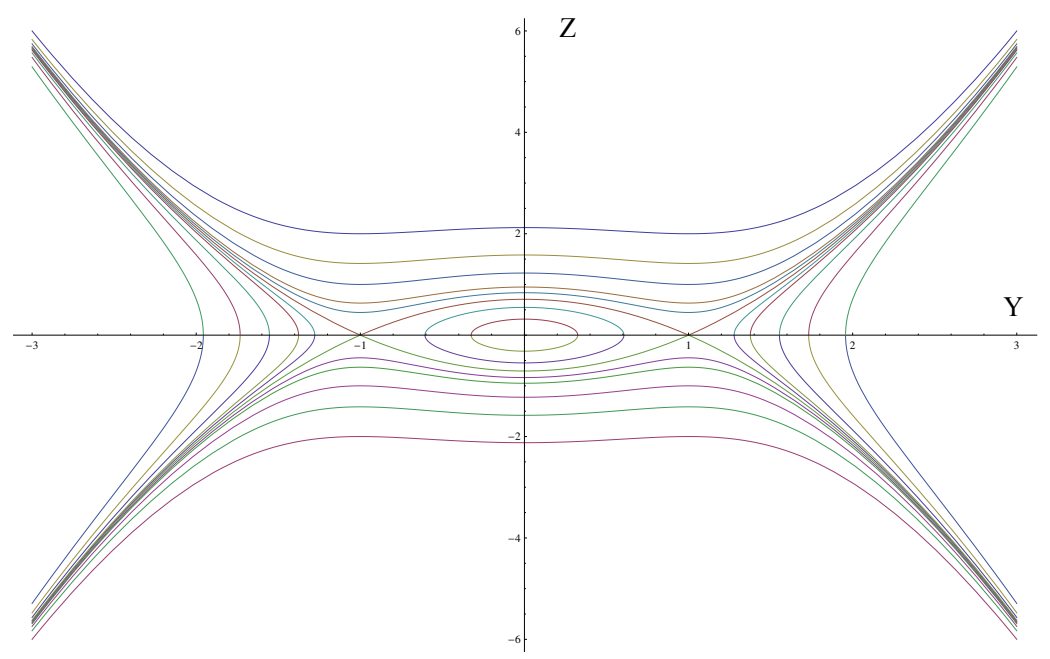

Figure 3. The orbits of the Hamiltonian systems (2.8) with $\epsilon=\frac{1}{\sqrt{2}}$ and with several values for $H$. We observe that for $K=0$ the set of level curves is symmetric with respect to the vertical axis.

3. If the energy is negative and satisfies

$$
-\frac{1}{2 \sqrt{2} \varepsilon}<H<0
$$

there exists a family of periodic orbits inside the region $|Y|<1$ (Observe that the fixed point $(0,0)$ for the restricted system is a center).

Proof. The proof is a straightforward consequence of what we have observed above and can be rigorously proved using standard Hamiltonian methods.

In the sequel, we will provide a brief description of these kinds of solutions.

\subsection{Periodic Solutions}

We rewrite for simplicity the condition of existence of periodic solutions in the $Y Z$ plane in the following way:

$$
\left(Y^{2}-1\right)^{2}-2 Z^{2}=p^{2}
$$

where $p^{2}=-2 \sqrt{2} \varepsilon H \in(0,1)$.

Then we look for a periodic orbit $(Y, Z)$ that satisfies

$$
\left(\frac{Y^{2}-1}{p}\right)^{2}-2\left(\frac{Z}{p}\right)^{2}=1,
$$

with $p \in(-1,1)$ and $p \neq 0$. As a consequence we have: 
Remark 2.3. The maximum and minimum values of $(Y, Z)$ for the periodic orbits satisfy:

$$
Y_{M A X, M I N}= \pm \sqrt{1-\sqrt{2 \sqrt{2}|H|}}= \pm \sqrt{1-p}
$$

and

$$
Z_{M A X, M I N}= \pm \sqrt{\frac{1}{2}-\sqrt{2} \varepsilon|H|}= \pm \frac{1}{\sqrt{2}} \sqrt{1-p^{2}}
$$

Remark 2.4. Recalling that $|H|=O\left(\varepsilon^{-1}\right)$ and taking into account the change of variable (2.5), we have

$$
x_{2}=O(1), \quad x_{3}=O\left(\varepsilon^{-1}\right)
$$

which means

$$
\max u_{x}=O(1), \quad \max u_{x x}=O\left(\varepsilon^{-1}\right) .
$$

This is in complete accordance with similar results obtained in [5] for the functional

$$
F_{\varepsilon, \mu}(u, \bar{u} ; I)=\frac{1}{2} \varepsilon^{2} \int_{I} u_{x x}^{2} d x+\frac{1}{2} \int_{I} W\left(u_{x}\right) d x+\frac{\mu}{2 \varepsilon^{2}} \int_{I}(u-\bar{u})^{2} d x
$$

whose minimizers share many properties with solutions of (1.2) (see [5] for a detailed discussion).

Then, to obtain a parametrization of the periodic orbits, for any $p^{2} \in(0,1)$ we set:

$$
\left(\frac{Z}{p}\right)^{2}=\left(\frac{1-p^{2}}{2 p^{2}}\right) \sin ^{2} \theta, \quad Z= \pm \sqrt{\left(\frac{1-p^{2}}{2}\right)} \sin \theta .
$$

This expression is suggested by that of $Z_{M A X}$ obtained for $\theta=\frac{\pi}{2}$.

We substitute it in (2.11), yielding

$$
\left(Y^{2}-1\right)^{2}=p^{2}+\left(1-p^{2}\right) \sin ^{2} \theta, \quad Y= \pm \sqrt{1-\sqrt{p^{2}+\left(1-p^{2}\right) \sin ^{2} \theta}}
$$

which, in particular, satisfies the expression of $Y_{M A X}$ for $\theta=0$.

In figure 4 below we represent the parametrization for $p=\frac{1}{2}$.

It is possible to obtain an estimate of the time period of the orbits that corresponds to the wave length of microstructure. Using the first integral and the second equation of the system we can write

$$
\frac{d Y}{d t}=\frac{\sqrt{2}}{\varepsilon} Z= \pm \frac{\sqrt{2}}{\varepsilon} \sqrt{\frac{1}{2}\left(Y^{2}-1\right)^{2}-\sqrt{2} \varepsilon|H|} .
$$

Then, if we integrate it between $\left(Y_{M I N}, Y_{M A X}\right)$, we obtain the spatial period $T_{H}=2 \varepsilon \int_{Y_{M I N}}^{Y_{M A X}} \frac{1}{\sqrt{\left(Y^{2}-1\right)^{2}-2 \sqrt{2} \varepsilon|H|}} d Y=2 \varepsilon \int_{Y_{M I N}}^{Y_{M A X}} \frac{1}{\sqrt{\left(Y^{2}-1\right)^{2}-p^{2}}} d Y$,

where the integrand does not depend on $\varepsilon$, indeed $p=O(1)$ since $|H|=$ $O\left(\varepsilon^{-1}\right)$ (see [2] for a discussion of the case $\varepsilon \rightarrow 0^{+}$).

We therefore have: 


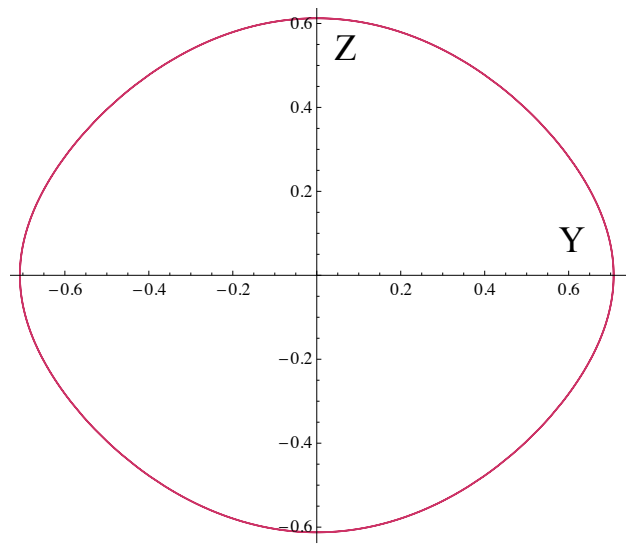

FiguRE 4. The parametrization of a periodic solution with $p=\frac{1}{2}$.

Proposition 2.5. For $H \in\left(-\frac{1}{2 \sqrt{2} \varepsilon}, 0\right)$, the spatial period satisfies

$$
T_{H}=O(\varepsilon),
$$

(as suggested in [1]).

For the energy value $H=-\frac{1}{2 \sqrt{2} \varepsilon}$ there exists the fixed point $P=(0,0,0)$ and then $T_{H}=0$, while if $H=0$, there are "heteroclinic connections" and henceforth $T_{H}=\infty$.

Finally we obtain the expression of $X$ as a function of $Y$ :

$$
\frac{d X}{d Y}=\frac{Y \frac{\varepsilon}{\sqrt{2}}}{ \pm \sqrt{\frac{1}{2}\left(Y^{2}-1\right)^{2}-\sqrt{2} \varepsilon|H|}},
$$

from which

$$
\begin{aligned}
X(Y) & = \pm \frac{\varepsilon}{\sqrt{2}} \int \frac{Y}{\sqrt{\frac{1}{2}\left(Y^{2}-1\right)^{2}-\sqrt{2} \varepsilon|H|}} d Y+C \\
& = \pm \frac{\varepsilon}{2} \int \frac{1}{\sqrt{Q^{2}-1}} d Q+C \\
& = \pm \frac{\varepsilon}{\sqrt{2}} \log \left|Q+\sqrt{Q^{2}-1}\right|+\omega
\end{aligned}
$$

where $\omega$ is a constant and

$$
Q=\frac{Y^{2}-1}{\sqrt{2 \sqrt{2} \varepsilon|H|}} .
$$

We note that $\max X=O(\varepsilon)$, that is $\max u=O(\varepsilon)$ (see also [5]).

The solution $X(Y)$ is represented in figure 5 below for fixed values of all the involved parameters. 


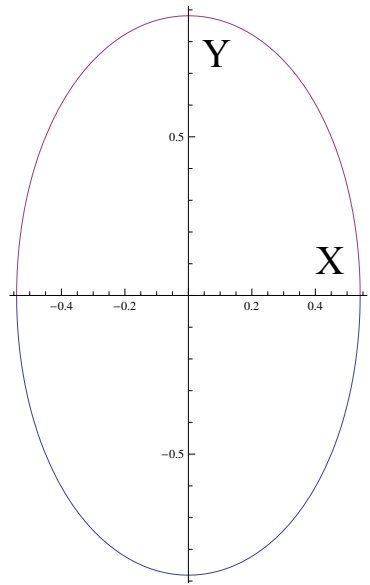

Figure 5. The function $X(Y)$ for $H=-\frac{1}{4 \sqrt{2} \varepsilon}$ in the plane $X Y$ with $\omega=0$ and $\varepsilon=\frac{1}{\sqrt{2}}$.

We remark that stationary periodic solutions may be considered as a good model for the description of microstructure formation.

\subsection{Unbounded Solutions}

Using hyperbolic functions it is possible to write $u(x)$ for $H>0$, that is, from the above computation:

$$
e^{ \pm \frac{\sqrt{2}}{\varepsilon}(X-\omega)}=Q+\sqrt{Q^{2}-1}
$$

and the following choice

$$
Q=\cosh \left(\frac{\sqrt{2}}{\varepsilon}(X-\omega)\right),
$$

satisfies the previous expression. For simplicity we set $u_{x}=u^{\prime}$, then

$$
\frac{\left(u^{\prime}\right)^{2}-1}{\sqrt{2 \sqrt{2} \varepsilon|H|}}=\cosh \left(\frac{\sqrt{2}}{\varepsilon}(u-\omega)\right) \text {. }
$$

In order to understand the shape of the solution, let us fix the constants $\omega=0, \varepsilon=\sqrt{2}, H=\frac{1}{4}$. Then:

$$
\left(u^{\prime}\right)^{2}=\cosh u+1=\cosh ^{2}\left(\frac{u}{2}\right) \text {, }
$$

and

$$
u^{\prime}= \pm \cosh \left(\frac{u}{2}\right)
$$

Finally, integrating, we deduce the expression of $u(x)$ :

$$
u(x)=4 \operatorname{arctanh}\left[\tan \left( \pm \frac{x}{4}\right)\right]+\text { const } .
$$


All the unbounded solutions, (that correspond to values $H>0$ ) have approximately the same shape and, as a consequence, the previous function qualitatively describes the whole class of such solutions (see figure 6 below).

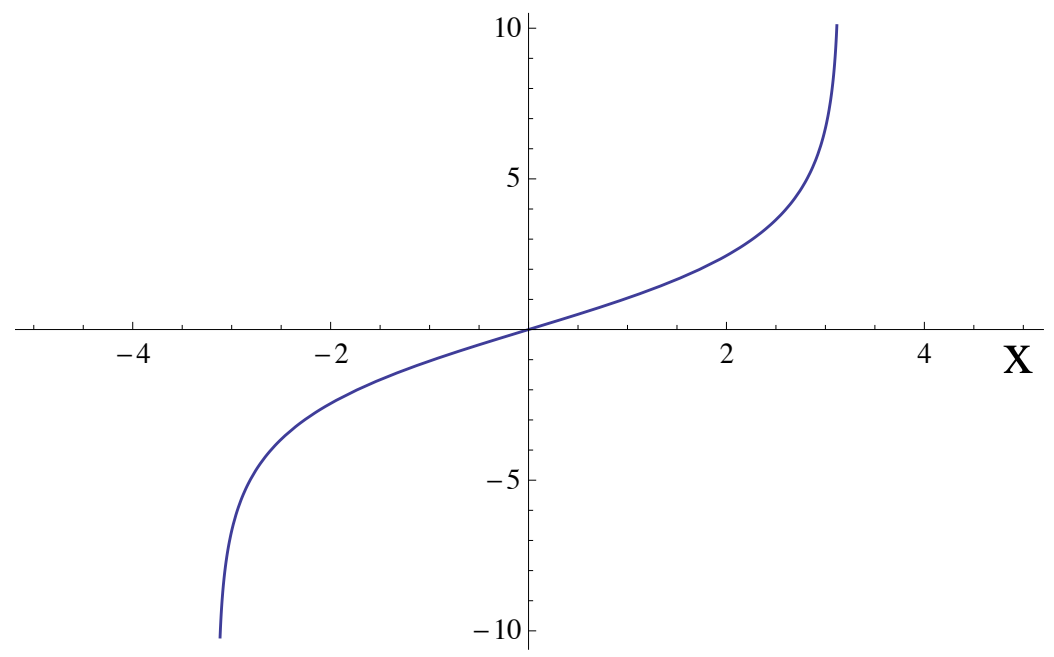

Figure 6. The unbounded solution with const $=0$.

\section{Traveling wave analysis}

In this section we deal with traveling wave solutions. We will see that the corresponding system of ODE's admit solutions that go to $\pm \infty$ eventually. However we will be able to extract some interesting informations for the qualitative behavior of the general solutions.

In particular, we look for traveling wave solutions of the following form

$$
u(x, t)=\varphi(x-c t)=\varphi(\xi) .
$$

Then

$$
u_{t}=-c \varphi^{\prime}(\xi), \quad u_{x}=\varphi^{\prime}(\xi)
$$

and the equation takes the form

$$
-c \varphi^{\prime}(\xi)+\varepsilon^{2} \varphi^{(I V)}(\xi)=\frac{1}{2}\left[W^{\prime}\left(\varphi^{\prime}(\xi)\right)\right]_{\xi},
$$

where all the derivatives are computed with respect to the variable $\xi$. Integrating (3.2) we obtain

$$
-c \varphi(\xi)+\varepsilon^{2} \varphi^{(I I I)}(\xi)=\frac{1}{2} W^{\prime}\left(\varphi^{\prime}(\xi)\right)+K,
$$

i.e.,

$$
\varepsilon^{2} \varphi^{(I I I)}(\xi)=c \varphi(\xi)+2 \varphi^{\prime}(\xi)\left[\left(\varphi^{\prime}(\xi)\right)^{2}-1\right]+K .
$$


As usual, we rewrite the above third order ordinary differential equation as a first order three dimensional system by using the following identification

$$
\begin{aligned}
& \varphi(\xi)=x_{1}, \quad \varphi^{\prime}(\xi)=x_{2}, \quad \varphi^{\prime \prime}(\xi)=x_{3}, \\
& \left\{\begin{array}{l}
\dot{x_{1}}=x_{2}, \\
\dot{x_{2}}=x_{3}, \\
\dot{x_{3}}=\frac{1}{\varepsilon^{2}}\left\{c x_{1}+2 x_{2}\left(x_{2}^{2}-1\right)+K\right\} .
\end{array}\right.
\end{aligned}
$$

Since we are considering the case $c \neq 0$, system (3.5) only possesses one fixed point:

$$
P=\left(-\frac{K}{c}, 0,0\right) \text {. }
$$

The Jacobian of the system at $P$ is

$$
J=\left(\begin{array}{ccc}
0 & 1 & 0 \\
0 & 0 & 1 \\
\frac{c}{\varepsilon^{2}} & -\frac{2}{\varepsilon^{2}} & 0
\end{array}\right)
$$

and the trace and determinant are

$$
\operatorname{Tr}(J)=0, \quad \operatorname{Det}(J)=\frac{c}{\varepsilon^{2}} .
$$

The functional Jacobian, as well as its trace and determinant, do not depend on $K$, then without loosing generality we consider the case

$$
K=0 .
$$

As a consequence, the system admits the fixed point $P=(0,0,0)$ for any value of $c$.

Then it appears natural to look for solitons, that is, solutions of (3.5) such that

$$
\lim _{\xi \rightarrow \pm \infty}\left(\varphi(\xi), \varphi^{\prime}(\xi), \varphi^{\prime \prime}(\xi)\right)=(0,0,0) .
$$

The task of proving the existence (or non existence) of such solutions can be very hard and perhaps misleading since our main interest is to find solutions which can reproduce the rich phenomenology described above (see introduction). In any case, numerical experiments (see below) suggest the existence of an unstable homoclinic cycle at $(0,0,0)$. The rigorous proof of this conjecture, even if it is far from the scope of the present article, could be of mathematical interest and will be analyzed in future works.

Then, in the sequel we will put aside condition (3.6) and study the solutions of system (3.5).

The characteristic polynomial of the Jacobian at $P$ is

$$
\lambda^{3}+\frac{2}{\varepsilon^{2}} \lambda^{2}-\frac{c}{\varepsilon^{2}}=0,
$$

with roots

$$
\lambda_{1}=U+V, \quad \lambda_{2,3}=-\frac{1}{2}(U+V) \pm i \frac{\sqrt{3}}{2}(U-V),
$$


where

$$
U=\sqrt[3]{\frac{c}{2 \varepsilon^{2}}+\sqrt{\frac{c^{2}}{4 \varepsilon^{4}}+\frac{8}{27 \varepsilon^{6}}}}
$$

and

$$
V=\sqrt[3]{\frac{c}{2 \varepsilon^{2}}-\sqrt{\frac{c^{2}}{4 \varepsilon^{4}}+\frac{8}{27 \varepsilon^{6}}}} .
$$

where $U, V \in \mathbb{R}$.

We have that

$$
U+V \geq 0, \quad \text { if and only if } \quad c \geq 0 .
$$

Then we have three cases:

$\diamond c<0$

$\diamond c=0$

$$
\lambda_{1}<0, \quad \operatorname{Re}\left(\lambda_{2,3}\right)=-\frac{\lambda_{1}}{2}>0 .
$$

$$
\lambda_{1}=0, \quad \operatorname{Re}\left(\lambda_{2,3}\right)=0, \quad \operatorname{Im}\left(\lambda_{2,3}\right)=\frac{\sqrt{2}}{\varepsilon} .
$$

$\diamond c>0$

$$
\lambda_{1}>0, \quad \operatorname{Re}\left(\lambda_{2,3}\right)=-\frac{\lambda_{1}}{2}<0 .
$$

When $c$ passes through the value zero, the real eigenvalue becomes positive and the real part of the complex conjugated eigenvalues become negative. Numerical simulations show that solutions remain close to a periodic orbit (or possibly an homoclinic cycle) and then, after a long time, start to move away. In figure 7 below we represent the solution in the time interval $\left(0, \varepsilon^{-2}\right)$ which is characterized by microstructure formation, and in the interval $\left(0,5 \varepsilon^{-2}\right)$ in which the coarsening phenomenon is observed. In figure 8 we represent the time series of $X(t)$ for a time of order $O\left(\varepsilon^{-2}\right)$ and $O\left(\varepsilon^{-3}\right)$ respectively. In the first case we observe oscillations of $u_{x}$ due to the phenomenon of microstructure formation while on the second one, we observe that the number of oscillations of $u_{x}$ decreases due to the coarsening process and $u_{x}$ converges to \pm 1 . Since we are considering the equation on the whole real line, if $\lim _{x \rightarrow \infty} u_{x}= \pm 1$ then $\lim _{x \rightarrow \infty} u= \pm \infty$.

In order to present some description of this phenomenon we rewrite $\dot{x_{3}}=\frac{1}{\varepsilon^{2}} f\left(x_{1}, x_{2}\right)$. Then it is easy to prove:

Proposition 3.1. The two sets

$$
A^{+}:=\left\{\left(x_{1}, x_{2}, x_{3}\right) \in \mathbb{R}^{3}: \quad f\left(x_{1}, x_{2}\right)>0, x_{2}>1, x_{3}>0\right\},
$$

and

$$
A^{-}:=\left\{\left(x_{1}, x_{2}, x_{3}\right) \in \mathbb{R}^{3}: \quad f\left(x_{1}, x_{2}\right)<0, x_{2}<-1, x_{3}<0\right\},
$$

are positively invariant for the system (3.5) with $c>0$. Moreover, any solution starting inside $A^{ \pm}$is unbounded, that is, $x_{1} \rightarrow \pm \infty$. 

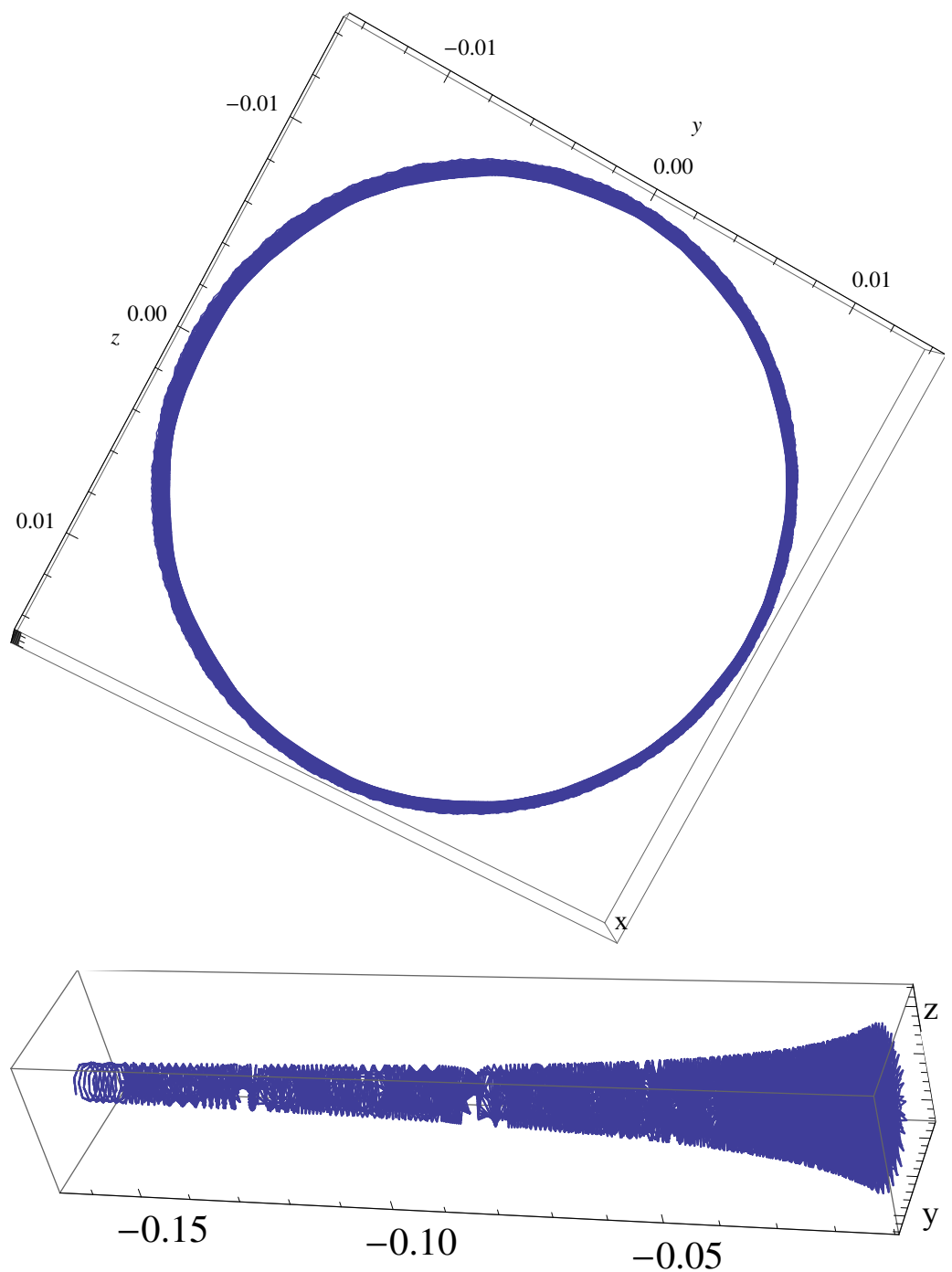

Figure 7. The solution of the system (3.5) in the interval $\left(0, \varepsilon^{-2}\right)$ and $\left(0,5 \varepsilon^{-2}\right)$ respectively.

For example in $A^{+}$we have

$$
\begin{aligned}
& \dot{x_{1}}+\dot{x_{2}}+\dot{x_{3}}=\frac{c}{\varepsilon^{2}} x_{1}+x_{2}+x_{3}+\frac{2}{\varepsilon^{2}} x_{2}\left(x_{2}^{2}-1\right) \\
& \geq \frac{c}{\varepsilon^{2}} x_{1}+x_{2}+x_{3} \\
& \geq \min \left\{1, \frac{c}{\varepsilon^{2}}\right\}\left(x_{1}+x_{2}+x_{3}\right) .
\end{aligned}
$$



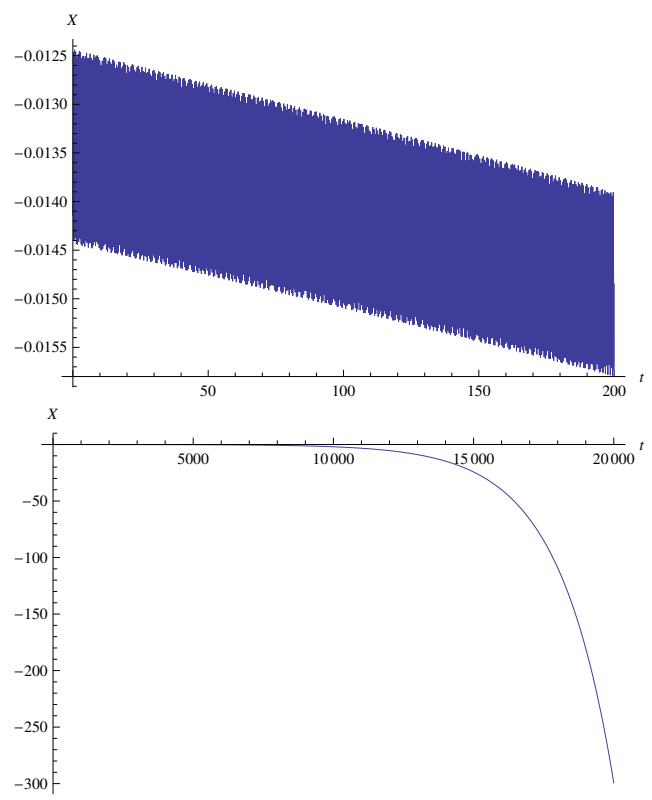

Figure 8 . The time series of $X(t)$ in a interval of time of order $O\left(\varepsilon^{-2}\right)$ and $O\left(\varepsilon^{-3}\right)$ respectively: in the first case we observe oscillations while in the second $u_{x} \rightarrow-1$.

Moreover, since $\dot{x_{1}}>0$, there exists a time $T$ at which $x_{1}(T)+x_{2}(T)+x_{3}(T)$ becomes positive and from the precedent inequality we obtain that $x_{1}+x_{2}+x_{3}$ explodes with $x_{1}, x_{2}, x_{3} \geq 0$.

Moreover, we have the following:

Theorem 3.2. $\quad$ 1. If a solution starting outside $A^{ \pm}$reaches the point $(0, \pm 1,0)$ at some time, then it enters $A^{ \pm}$.

2. Solutions starting inside

$$
B^{+}=\left\{(x, y, z) \in \mathbb{R}^{3}: \quad f(x, y)>0, \quad y \geq \frac{\sqrt{3}}{3}, \quad z>0\right\},
$$

enter $A^{+}$(a similar result is true for $B^{-}$and $A^{-}$) eventually.

3. For solutions in $B^{ \pm}$, if $x_{1} \rightarrow \pm \infty$ then $x_{2}, x_{3} \rightarrow \pm \infty$ and viceversa.

Proof. The proof of (1) is trivial, then we pass to provide just a few details for the proof of (2). If $x_{2} \geq \frac{\sqrt{3}}{3}$, then the function $g\left(x_{2}\right)=2 x_{2}\left(x_{2}^{2}-1\right)$ is increasing in $B^{+}$because $g^{\prime}\left(x_{2}\right)=2 x_{3}\left(3 x_{2}^{2}-1\right)$.

For the proof of (3) we note that from item (2) we have that solutions starting in $B^{+}$enter the positively invariant set $A^{+}$eventually, then on this set we have that $x_{1} \rightarrow+\infty$, from the third equation we have that $\dot{x}_{3} \rightarrow+\infty$ (since $x_{2}>1$ cannot diverge to $\left.-\infty\right)$ and as a consequence $x_{3} \rightarrow+\infty\left(\right.$ since $x_{3}>0$ 
in $A^{+}$). From the second equation we have that $\dot{x}_{2} \rightarrow+\infty$ which implies that $x_{2} \rightarrow+\infty$ (since $x_{2}>1$ in $A^{+}$). A similar result is valid with $-\infty$ replacing $+\infty$ and $A^{-}$replacing $A^{+}$. The converse is also true since when solution enters $A^{+}$we have, by the first equation of the system, $\dot{x_{1}} \rightarrow \infty$ and as a consequence $x_{1} \rightarrow \infty$.

From the previous results we have obtained several classes of unbounded solutions. In the next section we will focus on a particular class of such solutions.

\section{Approximate Solutions}

In this section we construct an approximate solution of equation (3.3) with $K=0$ in order to qualitatively describe the dynamical features observed in the previous sections.

Let us write system $(3.5)$ with $c \neq 0$ in the coordinates $(X, Y, Z)$ :

$$
\left(\begin{array}{c}
\dot{X} \\
\dot{Y} \\
\dot{Z}
\end{array}\right)=\left(\begin{array}{ccc}
0 & 1 & 0 \\
0 & 0 & \frac{\sqrt{2}}{\varepsilon} \\
\frac{c}{\sqrt{2} \varepsilon} & -\frac{\sqrt{2}}{\varepsilon} & 0
\end{array}\right)\left(\begin{array}{c}
X \\
Y \\
Z
\end{array}\right)+\left(\begin{array}{c}
0 \\
0 \\
\frac{\sqrt{2}}{\varepsilon} Y^{3}
\end{array}\right) .
$$

In order to use perturbation theory we consider the following change of variable:

$$
X=\varepsilon \bar{X}, \quad Y=\varepsilon \bar{Y}, \quad Z=\varepsilon \bar{Z}, \quad c=\varepsilon \bar{c},
$$

for any $\varepsilon \neq 0$. Then we obtain:

$$
\left\{\begin{aligned}
\dot{\bar{X}} & =\bar{Y} \\
\dot{\bar{Y}} & =\frac{\sqrt{2}}{\varepsilon} \bar{Z} \\
\dot{\bar{Z}} & =\frac{\bar{c} \varepsilon}{\sqrt{2} \varepsilon} \bar{X}+\frac{\sqrt{2}}{\varepsilon} \bar{Y}\left(\varepsilon^{2} \bar{Y}^{2}-1\right)
\end{aligned}\right.
$$

and, in order to simplify the computation, using the cylindrical coordinates, for $(X, Y, Z) \neq(0,0,0)$ we have:

$$
\left\{\begin{array}{l}
\bar{X}=\bar{X}, \\
\bar{Y}=R \sin \theta, \\
\bar{Z}=R \cos \theta,
\end{array}\right.
$$

we obtain the following system with respect to the variables $(\bar{X}, R, \theta)$ :

$$
\left\{\begin{array}{l}
\dot{\bar{X}}=R \sin \theta \\
\dot{R}=\frac{\cos \theta}{\sqrt{2} \varepsilon}\left\{\bar{c} \varepsilon \bar{X}+2 R^{3} \varepsilon^{2} \sin ^{3} \theta\right\} \\
\dot{\theta}=\frac{\sqrt{2}}{\varepsilon}-\frac{\sin \theta}{\sqrt{2} R \varepsilon}\left\{\bar{c} \varepsilon \bar{X}+2 R^{3} \varepsilon^{2} \sin ^{3} \theta\right\}
\end{array}\right.
$$

To simplify this system, we use $\theta$ as an independent variable. The system becomes

$$
\left\{\begin{aligned}
\frac{d \bar{X}}{d \theta} & =\frac{\sqrt{2} \varepsilon R^{2} \sin \theta}{2 R-h(\varepsilon) \sin \theta}:=F_{1}(\varepsilon), \\
\frac{d R}{d \theta} & =\frac{h(\varepsilon) R \cos \theta}{2 R-h(\varepsilon) \sin \theta}:=F_{2}(\varepsilon),
\end{aligned}\right.
$$


where we have set

$$
h(\varepsilon):=h(R, \theta, \bar{c}, \varepsilon)=\bar{c} \varepsilon \bar{X}+2 \varepsilon^{2} R^{3} \sin ^{3} \theta .
$$

For the next computations it is worth observing the following properties of the function $h(\cdot)$ :

$$
\begin{aligned}
& h(0)=0, \\
& h^{\prime}(\varepsilon)=\bar{c} \bar{X}+4 \varepsilon R^{3} \sin ^{3} \theta, \\
& h^{\prime}(0)=\bar{c} \bar{X}, \\
& h^{\prime \prime}(\varepsilon)=h^{\prime \prime}(0)=4 R^{3} \sin ^{3} \theta, \\
& h^{\prime \prime \prime}(\varepsilon)=h^{\prime \prime \prime}(0)=0 .
\end{aligned}
$$

Let us consider the Taylor expansion of the functions $F_{i}$ with respect to the parameter $\varepsilon$ :

$$
F_{i}(\varepsilon)=F_{i}(0)+\varepsilon F_{i}^{\prime}(0)+\frac{\varepsilon^{2}}{2} F_{i}^{\prime \prime}(0)+O\left(\varepsilon^{3}\right) .
$$

We first observe that $F_{i}(0)=0$ for $i=1,2$. In details, we have for $F_{1}(\cdot)$ :

$$
\begin{aligned}
& F_{1}^{\prime}(\varepsilon)=A(\theta) \cdot\left\{\frac{1}{2 R-h(\varepsilon) \sin \theta}+\frac{\varepsilon h^{\prime}(\varepsilon) \sin \theta}{[2 R-h(\varepsilon) \sin \theta]^{2}}\right\}, \\
& F_{1}^{\prime}(0)=\frac{A(\theta)}{2 R}=\frac{R \sin \theta}{\sqrt{2}}, \\
& F_{1}^{\prime \prime}(\varepsilon)=A(\theta) \sin \theta \cdot\left\{2 \frac{h^{\prime}(\varepsilon)}{[2 R-h(\varepsilon) \sin \theta]^{2}}+\varepsilon\left[\frac{h^{\prime \prime}(\varepsilon)}{[2 R-h(\varepsilon) \sin \theta]^{2}}\right.\right. \\
& \left.\left.+2 \sin \theta \frac{h^{\prime 2}(\varepsilon)}{[2 R-h(\varepsilon) \sin \theta]^{3}}\right]\right\}, \\
& F_{1}^{\prime \prime}(0)=2 A(\theta) \sin \theta \frac{h^{\prime}(0)}{4 R^{2}}=\frac{\bar{c}}{\sqrt{2}} \sin ^{2} \theta \bar{X}, \\
& F_{1}^{\prime \prime \prime}(\varepsilon)=A(\theta) \sin \theta \cdot\left\{2 \frac{h^{\prime \prime}(\varepsilon)}{[2 R-h(\varepsilon) \sin \theta]^{2}}+4 \frac{h^{\prime 2}(\varepsilon) \sin \theta}{[2 R-h(\varepsilon) \sin \theta]^{3}}\right. \\
& \left.+\left[\frac{h^{\prime \prime}(\varepsilon)}{[2 R-h(\varepsilon) \sin \theta]^{2}}+2 \sin \theta \frac{h^{2}(\varepsilon)}{\left[2 R-h^{2}(\varepsilon) \sin \theta\right]^{3}}\right]+\ldots\right\}, \\
& F_{1}^{\prime \prime \prime}(0)=3 \sqrt{2} R^{2} \sin { }^{3} \theta \cdot\left\{R \sin ^{2} \theta+\frac{\bar{c}^{2} \bar{X}^{2}}{4 R^{3}}\right\},
\end{aligned}
$$


where we have set for simplicity $A(\theta)=\sqrt{2} R^{2} \sin \theta$.

For $F_{2}(\cdot)$ we have:

$$
\begin{aligned}
& F_{2}^{\prime}(\varepsilon)=2 R B(\theta) \cdot \frac{h^{\prime}(\varepsilon)}{[2 R-h(\varepsilon) \sin \theta]^{2}}, \\
& F_{2}^{\prime}(0)=\frac{B(\theta) \bar{c} \bar{X}}{2 R}=\frac{\bar{c}}{2} \bar{X} \cos \theta \\
& F_{2}^{\prime \prime}(\varepsilon)=2 R B(\theta)\left\{\frac{h^{\prime \prime}(\varepsilon)}{[2 R-h(\varepsilon) \sin \theta]^{2}}+\frac{2\left[h^{\prime}(\varepsilon)\right]^{2} \sin \theta}{[2 R-h(\varepsilon) \sin \theta]^{3}}\right\}, \\
& F_{2}^{\prime \prime}(0)=\left\{2 R^{3} \sin ^{2} \theta+\frac{\bar{c}^{2} \bar{X}^{2}}{2 R}\right\} \cos \theta \sin \theta, \\
& F_{2}^{\prime \prime \prime}(\varepsilon)=12 R \sin \theta B(\theta)\left\{\frac{h^{\prime}(\varepsilon) h^{\prime \prime}(\varepsilon)}{[2 R-h(\varepsilon) \sin \theta]^{3}}+\frac{\sin \theta\left(h^{\prime}(\varepsilon)\right)^{3}}{[2 R-h(\varepsilon) \sin \theta]^{4}}\right\}, \\
& F_{2}^{\prime \prime \prime}(0)=12 \bar{c} \bar{X} R^{2} \sin ^{2} \theta \cos \theta\left\{\sin ^{2} \theta+\frac{\bar{c}^{2} \bar{X}^{2}}{16 R^{4}}\right\},
\end{aligned}
$$

where we have set for simplicity $B(\theta)=R \cos \theta$.

Then the system becomes:

$$
\left\{\begin{array}{l}
\frac{d \bar{X}}{d \theta}=\varepsilon \frac{R}{\sqrt{2}} \sin \theta+\frac{\varepsilon^{2}}{2} \frac{\bar{c}}{\sqrt{2}} \sin ^{2} \theta \bar{X}+\frac{\varepsilon^{3}}{6} 3 \sqrt{2} R^{2} \sin ^{3} \theta \cdot\left\{R \sin ^{2} \theta+\frac{\bar{c}^{2} \bar{X}^{2}}{4 R^{3}}\right\}+O\left(\varepsilon^{4}\right) \\
\frac{d R}{d \theta}=\varepsilon \frac{\bar{c}}{2} \cos \theta \bar{X}+\frac{\varepsilon^{2}}{2}\left\{2 R^{3} \sin ^{2} \theta+\frac{\bar{c}^{2} \bar{X}^{2}}{2 R}\right\} \cos \theta \sin \theta \\
+\frac{\varepsilon^{3}}{6} 12 \bar{c} \bar{X} R^{2} \sin ^{2} \theta \cos \theta\left\{\sin ^{2} \theta+\frac{\bar{c}^{2} \bar{X}^{2}}{16 R^{4}}\right\}+O\left(\varepsilon^{4}\right) .
\end{array}\right.
$$

We write, for $\varepsilon$ small, the solution of (4.4) in the following form:

$$
\left\{\begin{array}{l}
\bar{X}(\theta)=\bar{X}_{0}+\varepsilon H_{1}(\theta)+\frac{\varepsilon^{2}}{2} L_{1}(\theta)+\frac{\varepsilon^{3}}{6} M_{1}(\theta)+O\left(\varepsilon^{4}\right), \\
R(\theta)=R_{0}+\varepsilon H_{2}(\theta)+\frac{\varepsilon^{2}}{2} L_{2}(\theta)+\frac{\varepsilon^{3}}{6} M_{2}(\theta)+O\left(\varepsilon^{4}\right),
\end{array}\right.
$$

where $H_{i}$ and $L_{i}$ for $i=1,2$ are smooth functions of $\theta$ which do not depend on $\varepsilon$ with $H_{i}(0)=L_{i}(0)=0$ for $i=1,2$.

Then, differentiating with respect to $\theta$, we obtain

$$
\left\{\begin{aligned}
\frac{d \bar{X}}{d \theta} & =\varepsilon \frac{d H_{1}}{d \theta}+\frac{\varepsilon^{2}}{2} \frac{d L_{1}}{d \theta}+\frac{\varepsilon^{3}}{6} M_{1}(\theta)+O\left(\varepsilon^{4}\right), \\
\frac{d R}{d \theta} & =\varepsilon \frac{d H_{2}}{d \theta}+\frac{\varepsilon^{2}}{2} \frac{d L_{2}}{d \theta}+\frac{\varepsilon^{3}}{6} M_{2}(\theta)+O\left(\varepsilon^{4}\right),
\end{aligned}\right.
$$

while using equations of the system we have:

$$
\left\{\begin{array}{l}
\frac{d \bar{X}}{d \theta}=\varepsilon \frac{R_{0}}{\sqrt{2}} \sin \theta+\frac{\varepsilon^{2}}{2}\left\{\sqrt{2} \sin \theta H_{2}(\theta)+\frac{\bar{c}}{\sqrt{2}} \sin ^{2} \theta X_{0}\right\} \\
+\frac{\varepsilon^{3}}{6}\left\{\frac{3}{\sqrt{2}} L_{2}(\theta) \sin \theta+\frac{3}{\sqrt{2}} \bar{c} \sin ^{2} \theta H_{1}(\theta)+3 \sqrt{2} R_{0}^{2} \sin ^{3} \theta\left[R_{0} \sin ^{2} \theta+\frac{\bar{c}^{2} \bar{X}_{0}^{2}}{4 R_{0}^{3}}\right]\right\}+O\left(\varepsilon^{4}\right), \\
\frac{d R}{d \theta}=\varepsilon \frac{\bar{c}}{2} \cos \theta \bar{X}_{0}+\frac{\varepsilon^{2}}{2}\left\{\bar{c} \cos \theta H_{1}(\theta)+2 R_{0}^{3} \sin ^{3} \theta \cos \theta+\frac{\bar{c}^{2} \bar{X}_{0}^{2}}{2 R_{0}} \sin \theta \cos \theta\right\} \\
\frac{\varepsilon^{3}}{6}\{\ldots\}+O\left(\varepsilon^{4}\right) .
\end{array}\right.
$$


From the two previous expressions we obtain the derivatives of the functions $H_{i}$ :

$$
\left\{\begin{aligned}
\frac{d H_{1}}{d \theta} & =\frac{R_{0}}{\sqrt{2}} \sin \theta \\
\frac{d H_{2}}{d \theta} & =\frac{\bar{c}}{2} \cos \theta \bar{X}_{0} .
\end{aligned}\right.
$$

Integrating the previous equations in $[0, \theta]$ we obtain the expression of the functions $H_{i}(\cdot)$ :

$$
\begin{aligned}
& H_{1}(\theta)=\frac{R_{0}}{\sqrt{2}}(1-\cos \theta), \\
& H_{2}(\theta)=\frac{\bar{c}}{2} \bar{X}_{0} \cos \theta .
\end{aligned}
$$

For the functions $L_{i}(\cdot)$ we have the following:

$$
\left\{\begin{aligned}
\frac{d L_{1}}{d \theta} & =\frac{\bar{c}}{\sqrt{2}} \bar{X}_{0}\left[\sin \theta \cos \theta+\sin ^{2} \theta\right], \\
\frac{d L_{2}}{d \theta} & =\frac{\bar{c} R_{0}}{\sqrt{2}}\left(\cos \theta-\cos ^{2} \theta\right)+2 R_{0}^{3} \sin ^{3} \theta \cos \theta+\frac{\bar{c}^{2} \bar{X}_{0}^{2}}{2 R_{0}} \sin \theta \cos \theta,
\end{aligned}\right.
$$

from which

$$
\left\{\begin{array}{l}
L_{1}(\theta)=\frac{\bar{c}}{2 \sqrt{2}} \bar{X}_{0}\left[\sin ^{2} \theta+\theta-\sin \theta \cos \theta\right], \\
L_{2}(\theta)=\frac{\bar{c} R_{0}}{\sqrt{2}}\left(\sin \theta-\frac{\theta}{2}-\frac{1}{4} \sin 2 \theta\right)+\frac{R_{0}^{3}}{2} \sin ^{4} \theta+\frac{\bar{c}^{2} \bar{X}_{0}^{2}}{4 R_{0}} \sin ^{2} \theta .
\end{array}\right.
$$

Finally, since we are only interested in the $\mathrm{X}$ component, we need to integrate the following

$$
\begin{aligned}
& \frac{d M_{1}(\theta)}{d \theta}=\frac{3}{\sqrt{2}}\left\{\frac{\bar{c} R_{0}}{\sqrt{2}}\left(\sin \theta-\frac{\theta}{2}-\frac{1}{4} \sin 2 \theta\right)+\frac{R_{0}^{3}}{2} \sin ^{4} \theta+\frac{\bar{c}^{2} \bar{X}_{0}^{2}}{4 R_{0}} \sin ^{2} \theta\right\} \sin \theta \\
& +\frac{3}{2} R_{0} \bar{c} \sin ^{2} \theta(1-\cos \theta)+3 \sqrt{2} R_{0}^{2} \sin ^{3} \theta\left[R_{0} \sin ^{2} \theta+\frac{\bar{c}^{2} \bar{X}_{0}^{2}}{4 R_{0}^{3}}\right],
\end{aligned}
$$

from which

$$
\begin{aligned}
M_{1}(\theta) & =\frac{3}{4} \bar{c} R_{0} \theta\{1+\cos \theta\}-\frac{3}{2} \bar{c} R_{0} \sin \theta \cos \theta-\frac{3}{4} \bar{c} R_{0} \sin \theta-\frac{3}{4} \bar{c} R_{0} \sin ^{3} \theta \\
& +\frac{15}{4} \sqrt{2} R_{0}^{3}\left\{-\frac{\sin ^{4} \theta \cos \theta}{5}-\frac{4}{15} \sin ^{2} \theta \cos \theta+\frac{8}{15}-\frac{8}{15} \cos \theta\right\} \\
& +\frac{9}{8} \sqrt{2} \frac{\bar{c}^{2} \bar{X}_{0}^{2}}{R_{0}}\left\{-\frac{\sin ^{2} \theta \cos \theta}{3}+\frac{2}{3}(1-\cos \theta)\right\} \\
& :=\bar{c} R_{0} \theta P_{1}(\theta)+\bar{c} R_{0} P_{2}(\theta)+R_{0}^{3} P_{3}(\theta)+\frac{\bar{c}^{2} \bar{X}_{0}^{2}}{R_{0}} P_{4}(\theta),
\end{aligned}
$$

where $P_{i}(\theta), i=1,2,3,4$, are bounded periodic functions.

Then we can write the approximated solution as

$$
\begin{aligned}
& \bar{X}(\theta)=\bar{X}_{0}+\varepsilon \frac{R_{0}}{\sqrt{2}}[1-\cos \theta]+\varepsilon^{2} \frac{\bar{c}}{4 \sqrt{2}} \bar{X}_{0}\left[\sin ^{2} \theta+\theta-\sin \theta \cos \theta\right] \\
& +\frac{\varepsilon^{3}}{6}\left[\bar{c} R_{0} \theta P_{1}(\theta)+\bar{c} R_{0} P_{2}(\theta)+R_{0}^{3} P_{3}(\theta)+\frac{\bar{c}^{2} \bar{X}_{0}^{2}}{R_{0}} P_{4}(\theta)\right]+O\left(\varepsilon^{4}\right) .
\end{aligned}
$$


We observe that the coefficient of $\theta$ is

$$
\frac{\varepsilon^{2}}{4} \frac{\bar{c}}{\sqrt{2}}\left\{\bar{X}_{0}+\frac{\varepsilon}{\sqrt{2}} R_{0}(1+\cos \theta)\right\} .
$$

Then, if we choose such that

$$
\bar{X}_{0}+\frac{\varepsilon}{\sqrt{2}} R_{0}=0
$$

we have

$$
\begin{aligned}
& \bar{X}(\theta)=-\varepsilon \frac{R_{0}}{\sqrt{2}} \cos \theta+\varepsilon^{3} \frac{\bar{c} R_{0}}{8}\left[-\sin ^{2} \theta+\sin \theta \cos \theta\right] \\
& +\frac{\varepsilon^{3}}{6}\left[+\bar{c} R_{0} P_{2}(\theta)+R_{0}^{3} P_{3}(\theta)+\frac{\bar{c}^{2} \bar{X}_{0}^{2}}{R_{0}} P_{4}(\theta)\right]+\frac{\varepsilon^{4}}{8} \bar{c} R_{0} \theta \cos \theta+O\left(\varepsilon^{4}(4.8)\right.
\end{aligned}
$$

we observe that the biggest term (for large time) among the remaining terms of order $O\left(\varepsilon^{4}\right)$ has the following form

$$
\bar{c}^{2} \bar{X}_{0} f_{1}(\theta) \theta^{2}
$$

where $f_{1}(\theta)$ is a periodic function. Then we consider the following approximated function:

$$
\bar{X}^{\circ}(\theta)=-\varepsilon \frac{R_{0}}{\sqrt{2}} \cos \theta+\varepsilon^{3} f_{2}(\theta)+\frac{\varepsilon^{4}}{8} \bar{c} R_{0} \theta \cos \theta,
$$

we observe that the higher order term of the difference between $\bar{X}^{\circ}(\theta)$ and the solution of the system, for large time, can be estimated by

$$
\bar{D}(\theta)=k_{1}\left(\theta^{2}+k_{2}\right) R_{0} \varepsilon^{5} \bar{c}^{2} .
$$

Coming back to the original coordinates we have

$$
X^{\circ}(\theta)=\varepsilon \bar{X}^{\circ}(\theta)=-\varepsilon^{2} \frac{R_{0}}{\sqrt{2}} \cos \theta+\varepsilon^{4} f_{2}(\theta)+\frac{\varepsilon^{5}}{8} c R_{0} \theta \cos \theta,
$$

and the error

$$
D(\theta)=k_{1}\left(\theta^{2}+k_{2}\right) R_{0} \varepsilon^{7} c^{2} .
$$

Then for $\theta<\varepsilon^{3}$ the leading term of $X^{\circ}(\theta)$ is a periodic function:

$$
\gamma(\theta)=-\varepsilon^{2} \frac{R_{0}}{\sqrt{2}} \cos \theta
$$

while for time of order $\theta=O\left(\varepsilon^{3}\right)$ the function starts to loose periodicity. Using the third equation of the system in polar coordinates we obtain the relation between $\theta$ and the "original time" $t$ :

$$
\theta \approx \frac{\sqrt{2}}{\varepsilon} t
$$

The approximate solution qualitatively describes what was obtained by numerical experiments: the solutions appear to be periodic (microstructure formation) for a large interval of time and then start to loose periodicity (in the sense that a coarsening phenomenon occurs). In particular, when $\theta<<\varepsilon^{-3}$ (that is $t<<\varepsilon^{-2}$ ), the function (4.11) appears periodic, while when $\theta=O\left(\varepsilon^{-3}\right)$ (that is $t=O\left(\varepsilon^{2}\right)$ ) the linear term in $\theta$ cannot be disregard 
and the solutions "start to loose periodicity".

We conclude the present work with several numerical simulations to illustrate the results of the present section. In the figures 9 below we compare the solution $\bar{X}(\theta)$ with the periodic function $\bar{X}_{0}+\frac{\varepsilon}{\sqrt{2}} R_{0}[1-\cos \theta]$ in several time scales.
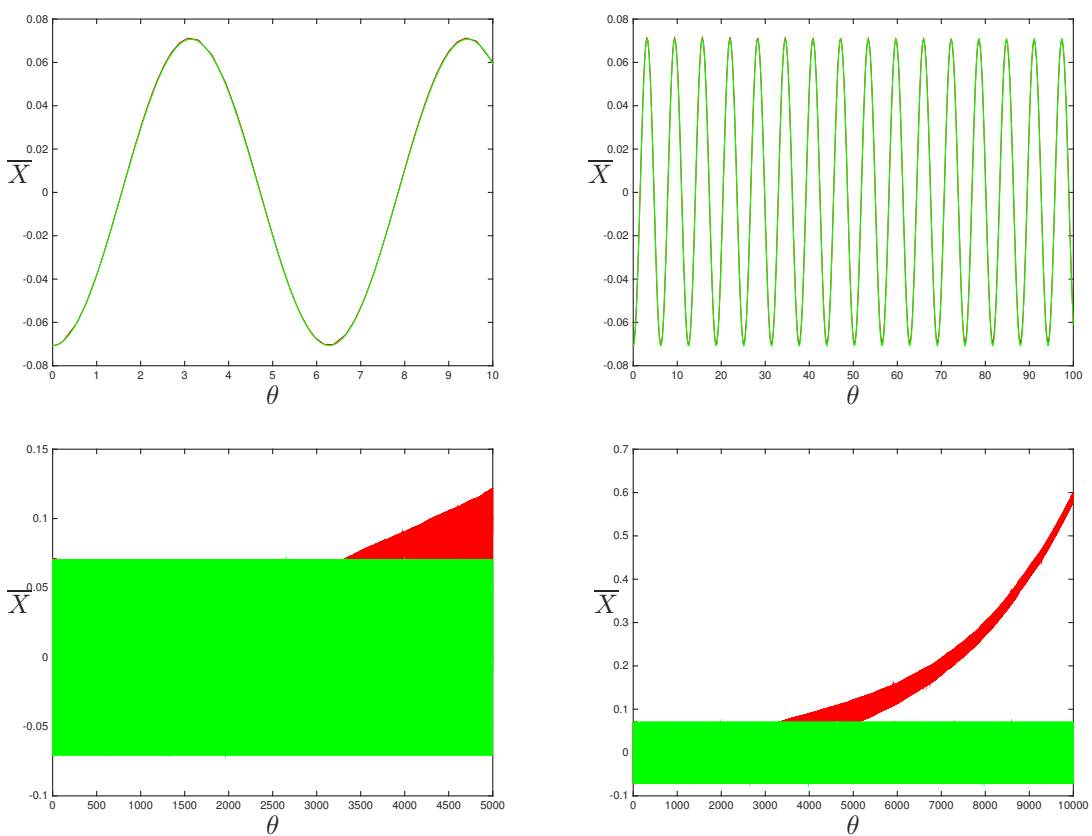

FiguRE 9. The comparison between the numerical solution $\bar{X}(\theta)$ (in red) computed using the Software Matlab and the function $\bar{X}_{0}+\frac{\varepsilon}{\sqrt{2}} R_{0}[1-\cos \theta]$ (in green) with $\varepsilon=\frac{1}{10}, R_{0}=1$, $\bar{X}_{0}=-\frac{R_{0}}{2} \varepsilon$ and $\bar{c}=0.1$. In the the first two simulations the time intervals are of order $O\left(\varepsilon^{-1}\right)$ and $O\left(\varepsilon^{-2}\right)$ respectively, in particular $[0,10]$ and $[0,100]$ respectively. The numerical solution and the function $\gamma(\theta)$ cannot be distinguished. In the remaining simulations, we consider a time of order $O\left(\varepsilon^{-3}\right)$, $[0,5000]$ and $O\left(\varepsilon^{-4}\right)$ and $[0,10000]$. We observe that in the latter case the solution is no more periodic while for a time of order $O\left(\varepsilon^{-3}\right)$ the solution becomes to move from the periodic approximation.

In figure 10 below we compare the solution $\bar{X}(t)$ of (4.2) with the function $\bar{X}_{0}+\frac{\varepsilon}{\sqrt{2}} R_{0}\left[1-\cos \left(\frac{\sqrt{2}}{\varepsilon} t\right)\right]$ in a time scale of order $t=O(\varepsilon)$. 


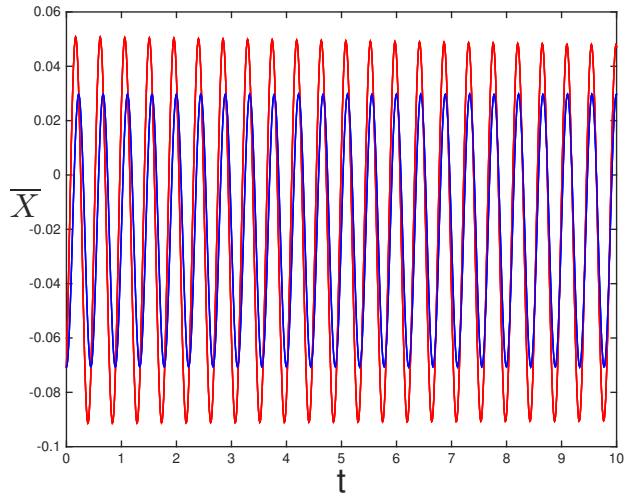

Figure 10. The solution $\bar{X}(t)$ of (4.2) (in red) and the function $\bar{X}_{0}+\frac{\varepsilon}{\sqrt{2}} R_{0}\left[1-\cos \left(\frac{\sqrt{2}}{\varepsilon} t\right)\right]$ in a time scale of order $t=$ $O(\varepsilon)$.

\section{Conclusion}

In this work we have presented a qualitative description of microstructure formation and coarsening phenomena for the solutions of a nonlinear equation presenting three different time scales with different dynamical features. Even if it can be considered only a preliminary work we consider that the present analysis could represent a good starting point for future research We have related the microstructure formation to the existence of periodic stationary solutions (analyzed in Section 2) while the coarsening phenomenon has been described by studying a class of unbounded solutions (Section 3 and Section 4). We leave for future research the problem of the complete description of the set of stationary solutions and the existence/non existence of solitons. Moreover, we consider that the coarsening phenomenon could be analyzed with different methods both numerically and analytically.

\section{Acknowledgements}

We would like to thank the anonymous referees for their interesting and very helpful suggestions that contributed to clarify the problem under consideration and to improve the quality of the paper.

\section{References}

[1] Bellettini, G.; Fusco, G.; Guglielmi, N. A concept of solution and numerical experiments for forward-backward diffusion equations. Discrete Continuous Dynamical Systems 16, no. 4, (2006), 783-842. 
[2] Bellettini G., Fusco, G. The $\Gamma$-limit and the related gradient flow for singular perturbation functionals of Perona-Malik type. Transactions of the American Mathematical Society, Volume 360, Issue 9, (2008), 4929-4987.

[3] Caraballo, T; Colucci, R. The effects of additive and multiplicative noise on the dynamics of a parabolic equation. Applied Mathematics \& Information Science, Volume 9, No. 5, (2015), 2273-2281.

[4] Caraballo, T; Colucci, R. Pullback attractor for a non-linear evolution equation in elasticity. Nonlinear Analysis: Real World Applications, Volume 15, (2014), 80-88.

[5] Colucci, R. Analysis of microstructure of a non-convex functional with penalization term. J. Math. Anal. Appl. 388, no. 1, (2012), 370-385.

[6] Colucci, R. Existence of global and blowup solutions for a singular second-order ODE. Electronic Journal of Differential Equations, 307, pp. 1 - 17. 2015.

[7] Colucci, R.; Chacón, G.R. Dimension Estimate for the Global Attractor of an evolution equation. Abstract and Applied Analysis, Article ID 541426, (2012), 18 pages, doi: $10.1155 / 2012 / 541426$.

[8] Colucci, R.; Chacón, G.R. Hyperbolic Relaxation of a Fourth Order Evolution Equation. Abstract and Applied Analysis, Article ID 372726, (2013), 11 pages http://dx.doi.org/10.1155/2013/372726

[9] Colucci, R.; Chacón, G.R. Asymptotic behavior of a fourth order evolution equation. Nonlinear analysis: Theory, Methods \& Applications, 95 (2014) 6676.

[10] Colucci R.; Chacón, G.R.; Rafeiro, H. \& Vargas Dominguez, A. On minimization of a non-convex functional in variable exponent spaces. International Journal of Mathematics, Vol. 25, No. 1, (2014), 1450011 (19 pages).

[11] Constantin, P.; Foias, C.; Nicolaenko, B.; Temam, R. Integral Manifolds and Inertial Manifolds for Dissipative Partial Differential Equations. Applied Mathematical Sciences v. 70, Springer; 1 edition, (1988).

[12] Demoulini, S. Young measure solutions for a nonlinear parabolic equation of forwardbackward type. SIAM J. Math. Anal. 27 (2) (1996).

[13] Eden, A.; Foias, C.; Nicolaenko, B.; Temam, R. Exponential Attractors for Dissipative Evolution Equations. John Wiley \& Sons Inc., (1995).

[14] Müller, S. Variational models for microstructure and phase transitions. Calculus of variations and geometric evolution problems (Cetraro, 1996), 85-210, (1999) Lecture Notes in Math., 1713, Springer, Berlin.

[15] Pedregal, P. Variational Methods in Nonlinear Elasticity. Society for Industrial and Applied Mathematics (SIAM), Philadelphia, PA, (2000).

[16] Robinson, J.C. Infinite-dimensional dynamical systems. An introduction to dissipative parabolic PDEs and the theory of global attractors. Cambridge Texts in Applied Mathematics. Cambridge University Press, Cambridge, (2001).

[17] Robinson, J.C. Dimensions, Embeddings, and Attractors. Cambridge Tracts in Mathematics. Cambridge University Press, Cambridge.

[18] Slemrod, M. Dynamics of measured valued solutions to a backward-forward heat equation. Journal of Dynamics and Differential Equations Volume 3, Number 1, (1991), 1-28.

[19] Temam, R. Infinite Dimensional Dynamical Systems in Mechanics and Physics. Springer; 2nd edition, (1997). 


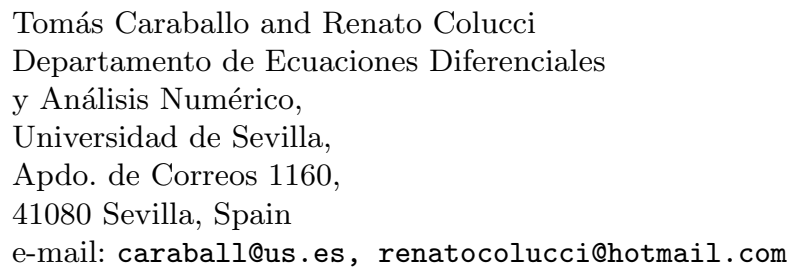

